\title{
Fault Diagnosis of Gearbox Based on Laser Particle Size Analysis
}

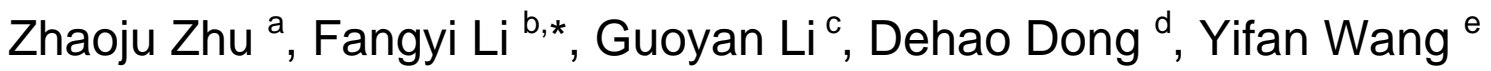 \\ Ministry of Education Key Laboratory of Efficient and Clean Machinery Manufacturing, School of \\ Mechanical Engineering, Shandong University, Jinan 250061, China \\ azhuzhaoju0216@163.com, blifangyi2006@qq.com, Cliguoyan821@qq.com, ddongdehao2014@qq. \\ com, ewangyifan102007@126.com
}

Keywords: Laser particle size; Fault diagnosis; Oil monitoring; Gearbox.

\begin{abstract}
This paper summarizes the working principle of laser particle size analyzer, and some experimental study can be made to monitor the number and size of abrasive particle in the oil of lubricant by the laser particle size analysis method. It obtains the particle size distribution of lubricant's abrasive particle at different times, so the working status of the gearbox can be judged by it. At the same time, we can sum up the growth law of the largest abrasive particle and the minimum abrasive particle in the lubricant. So we can judge the abnormal wear time of device to achieve timely repair and maintenance of equipment. The results showed that the laser particle size analysis method can quickly and efficiently monitor the particle size distribution of abrasive particle in the lubricant. This can provide the advantageous basis for judging the running state of the bulldozer's gearbox, consequently this can effectively prevent equipment failure.
\end{abstract}

\section{Introduction}

Currently, in order to ensure the scientific and efficient development of modern industry, it's increasingly demanding for working equipment's running, and equipment failure are generally on prevention, maintenance is complementary. In recent years, oil monitoring technology which is a new type of condition monitoring techniques are widely used in condition monitoring and fault prevention of construction machinery and other large mechanical equipment. The technique is the use of technical means of optical, electrical, magnetic, etc. And we can analyze the physical and chemical indicators, abrasive particle and external contaminants particles of equipment lubricants sample which collected. Consequently, we can obtain equipment's lubrication and abrasive particle information, and it can qualitatively and quantitatively describe wear condition of the device, evaluate the equipment conditions, predict the equipment failures, and these can be used to determine the location, cause and type of the fault[1]. Now there are several oil analysis methods commonly used, such as the physical and chemical properties analysis which can monitor the quality of lubricating oil, and iron spectral analysis, particle counting analysis and atomic spectroscopy analysis which can monitor the wear particles, etc. Tang Dexiu etal, made a study of automobile mechanical wear fault prediction by iron spectral analysis. He extracted the fault feature wear abrasive particle of vehicle power system, judged the location and degree of wear by the abrasive particle characteristics, and thus judged the mechanical operation state, finally made the mechanical fault prediction[2]; Zhou Xiaohong etal, monitored and analyzed the abrasive particle of aero-engine lubricating oil by the application of particle counting method, obtained the size and number of the abrasive particle by this method, judged the wear state of the engine, to make sure reliable operation of aviation engines[3]; Zheng Changsong etal, monitored the hydraulic lubricating oil of a tracked armored vehicles integrated drive, and analyzed the concentration of each abrasive particles in oil, provided theoretical and experimental basis for the realization of armored vehicles' maintenance as appropriate, improved the reliability and maintainability of integrated device[4].

Due to the abrasive particles size range widely distributed in oil, a separate iron spectral analysis or spectral analysis can't accurately measure the abrasive particles' content and size in oil, so this paper introduces a new type of oil analysis methods - laser particle size analysis. This technique use laser diffraction to measure abrasive particles' particle size distribution in oil, judged wear condition of mechanical equipment by analyzing the size of abrasive particles' particle size distribution. It has 
many advantages, such as easy operation, rapid measurement, good repeatability, wide measurement range etc. There is important application value for the fast implementation of key parts' remanufacture judgment.

\section{The theory of Laser Particle Size Analyzer}

Laser particle size analysis is based on the principle of laser diffraction [5], according to the difference of the scattering angle which formed by laser irradiation of different size abrasive particle, the small abrasive particle has large scattering angles by the laser, the large abrasive particle has small scattering angle by the laser, the size of the abrasive particle can be converted by measuring the scattering angle.Winner2005 laser particle size analyzer's works is showed in Figure 1.

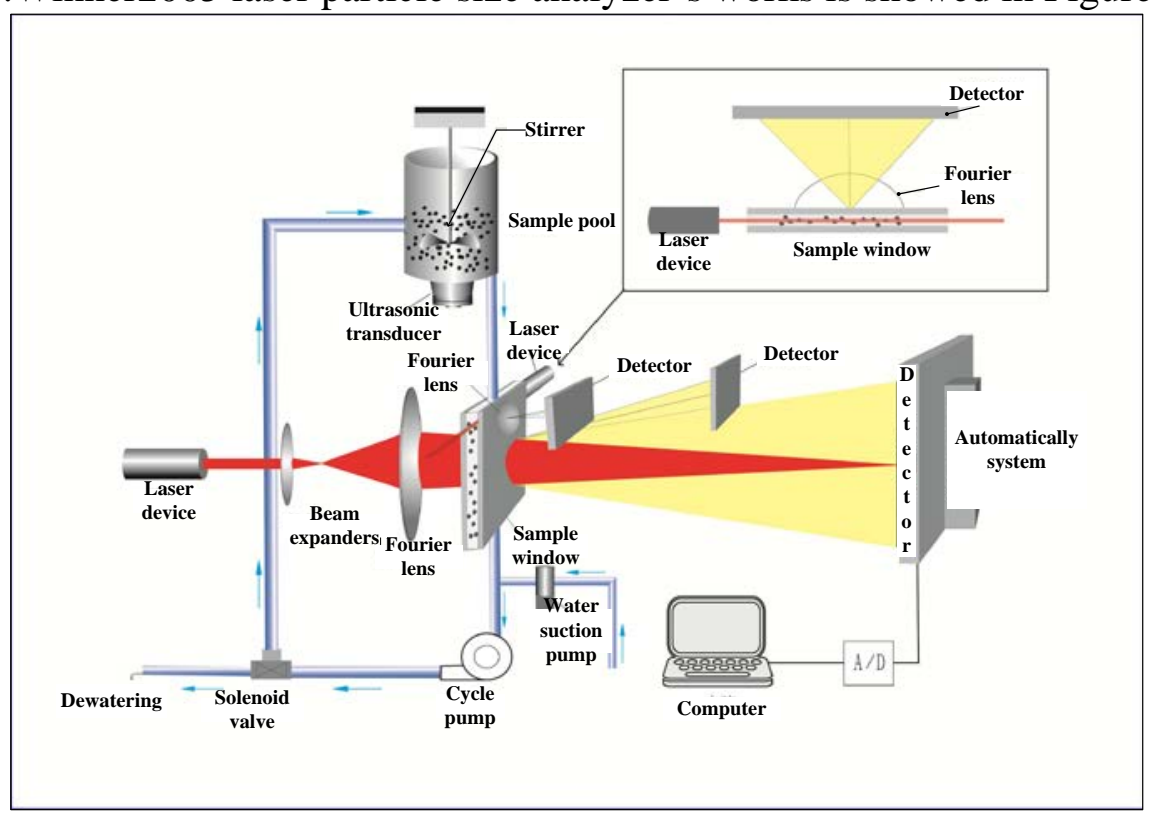

Fig.1 Winner2005 laser particle size analyzer working principle diagram

Monochromatic light emitted by the high duty He-Ne laser expand into a wider light beam through beam expander [6], when the light exposure to the abrasive particles of detected oil in the sample window after transforming through the Fourier mirror, abrasive particles of different sizes scattered light on the forward according to different angles, the scattering angle is close to the diffraction that pores which is the same as the diameter of the abrasive particles produced. The scattered light generated by the abrasive particles and then focused by a Fourier transform lens onto the detector of focal plane behind the lens, while the remaining incident light is not scattered, and it focus on the center of the main detector, not affecting the scattering of light. The light scattering information of abrasive particles that the main detector received is sent into the computer for processing after the A/D-converted, consequently we can obtain the particle size distribution information of abrasive particles in the detected oil.

The relationship between scattered light intensity of abrasive particles and the particle size can be showed by the formula (1) [7]:

$$
I(\theta)=\frac{1}{\theta} \int_{0}^{\infty} R^{2} \mathrm{n}(R) J(\theta K R) \mathrm{d} R
$$

In the above formula : $\theta$ is scattering angle; $R$ is the particle radius; $I(\theta)$ is the light intensity by angle scattering; $\mathrm{n}(R)$ is abrasive particle size distribution function; $J(\theta K R)$ is the first Bayesian function,among them $K=2 \pi / \lambda, \lambda$ is the laser wavelength. The function of the particle diameter distribution can be obtained by (1).

\section{Experimental Materials and Equipment}

The main experimental materials includes experimental oil samples which taken from the SD16 bulldozer gearbox. The gearbox is power shift and the model number of lubricant is 15W 40-CD. 
Each sampling interval is around $100 \mathrm{~h}$, a total of four samples, oil samples were numbered 1, 2, 3,4. The experimental instruments and equipment includes Winner2005 laser particle size analyzer, DHY-9075A-type electric blast oven, glass rods, rubber tubes, funnels, beakers and so on.

\section{Experimental results and analysis}

The abrasive particles contained in the oil sample can settle down to the bottom of the sampling bottle due to gravity action in the delivery process of the oil sample, leading the abrasive particles to the vertical uneven distribution in the sample bottle. In order to avoid the experimental error caused by the uneven distribution of the abrasive particles, standard metal chemistry within the lubricant is required before conducting experiments on oil samples were heated with stirring treatment, the oil sample need the heating and stirring processing before experiments. First of all, the sample should be heated in the DHY-9075A-type electric oven thermostat blast. According to metal chemical analysis standard methods [8] in lubricants of the American Society of Testing and Materials, the heating temperature is set to $65^{\circ} \mathrm{C} \pm 5^{\circ} \mathrm{C}$ and heat preservation around 30 minute,so that the lubricant of bottle is sufficiently formed convection. Then we remove oil sample from the dry box and shaking with the hand for at least 5 minutes to make the abrasive particles complete dispersion in the oil sample, we can do experiment after the abrasive particles showing the state of a homogeneous suspension.

The abrasive particles or particulate contaminants of the oil can cause the originals wearing and tearing, which reduces the working performance of the machinery, especially the transmission of construction machinery products, such as gearbox. The abrasive particles of oil will decrease the reliability of the transmission work, if that is not timely monitoring and clean up the abrasive particles of oil and other pollutants, it will increase the wear of parts inside the device with the increase of abrasive particles, and reduce the efficiency of the device, thus greatly shortening the life of the equipment. Therefore, the real-time monitoring of the number of abrasive particles is particularly important. We can respectively analyze the size of abrasive particles in oil sample 1,2,3,4 by the laser particle size analyzer, the corresponding experimental results obtained is in Figure2,3,4,5.

As you can see from Figure 2, the particle size of the abrasive particles in oil is a roughly normal distribution, the particle size mainly distribute between the $0 \sim 8 \mu \mathrm{m}$, the abrasive particles in this size range are normal and the function reaches the maximum when the particle size is about $2 \mu \mathrm{m}$. That is, the volume occupied by the abrasive particles is maximum when the particle size is about $2 \mu \mathrm{m}$, and there are no abnormal abrasive particles. It indicates that the machinery and equipment is in good state of normal wear and tear at this time, and it can work safely and reliably.

As you can see from Figure 3, the volume ratio occupied by the abrasive particles will first increase and then decrease with the increase of the particle diameter, and the function reaches the maximum when the particle size is about $3 \mu \mathrm{m}$. That is, the volume occupied by the abrasive particles is the maximum when the particle size is about $2 \mu \mathrm{m}$, compared with Figure 1, the average particle size of the abrasive particles has increased. But there are no abnormal abrasive particles or content mutant phenomenon. It still within the range of normal abrasive particles, the equipment wear state is in areas of normal wear and tear, and the equipment can continue normal operation.

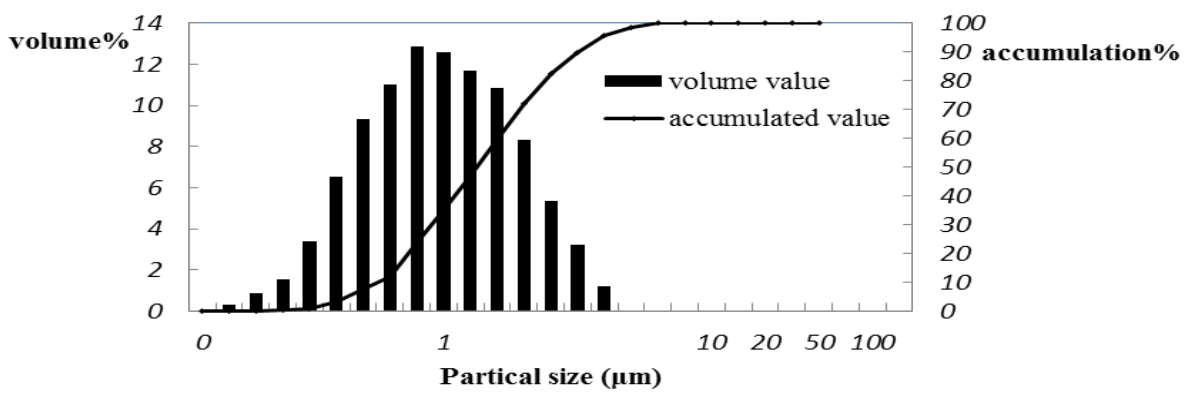

Fig. 2 The particle size distribution figure of the oil sample 1 


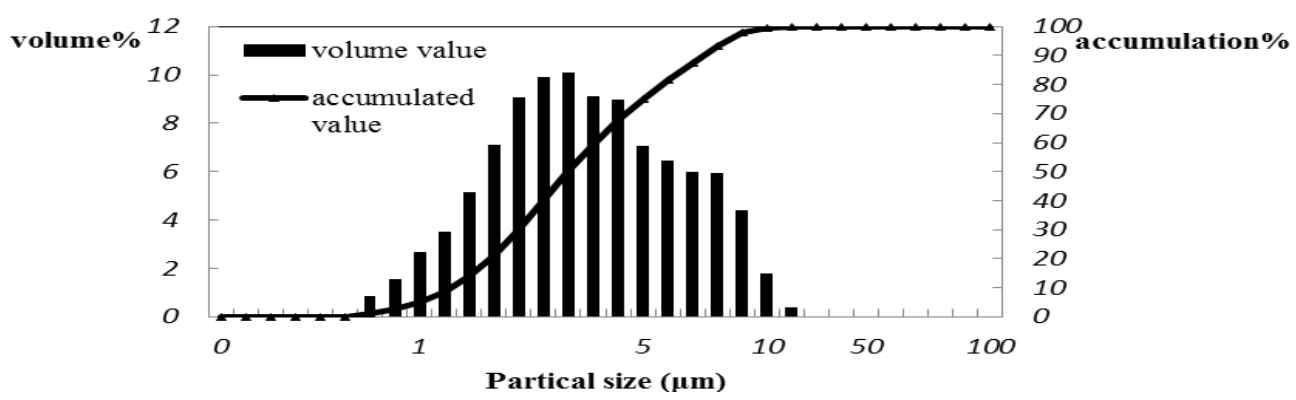

Fig. 3 The particle size distribution figure of the oil sample 2

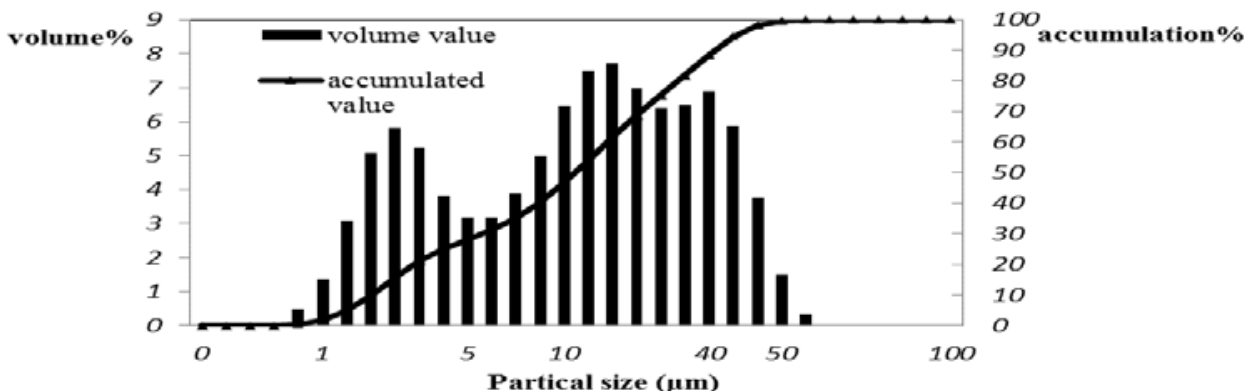

Fig. 4 The particle size distribution figure of the oil sample 3

As you can see from Figure 4, the maximum particle size of the abrasive particles is $52 \mu \mathrm{m}$, and the function reaches the maximum when the particle size is about $3 \mu \mathrm{m}$ and $12 \mu \mathrm{m}$. The volume occupied by the abrasive particles is the maximum when the particle size is about $12 \mu \mathrm{m}$, the particle size range of the abrasive particles becomes $0 \sim 52 \mu \mathrm{m}$, the particle size than figure 1 and figure 2 has increased. It indicates that the abrasive particle size is too large at this time, it's the omen of the abnormal wear of mechanical equipment, it is necessary to draw attention and strengthen the monitoring to equipment.

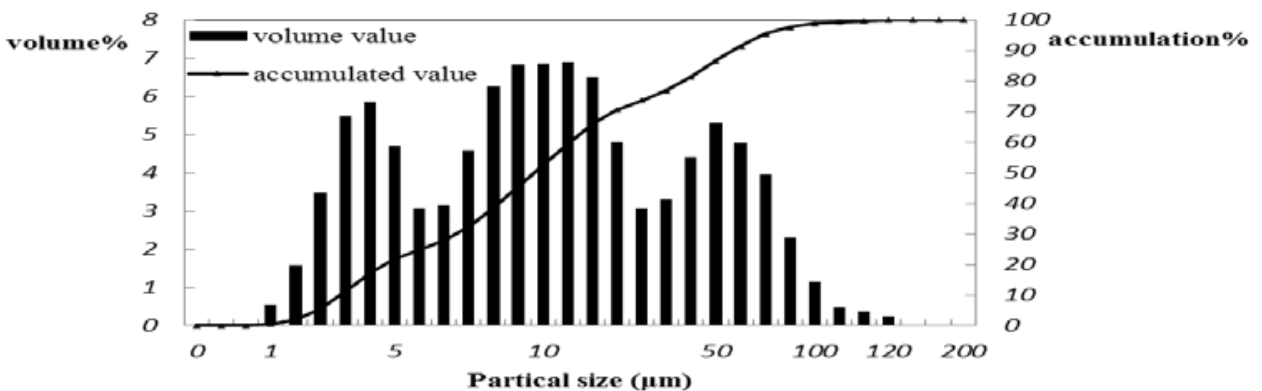

Fig. 5 The particle size distribution figure of the oil sample 4

As you can see from Figure 4, the particle size range of the abrasive particles in oil becomes 0 $120 \mu \mathrm{m}$, the maximum particle size of the abrasive particles is $121 \mu \mathrm{m}$ and the function in the Figure 4 reaches the maximum when the particle size is about $4 \mu \mathrm{m}, 11 \mu \mathrm{m}$ and $50 \mu \mathrm{m}$. The volume occupied by the abrasive particles is the maximum when the particle size is about $11 \mu \mathrm{m}$. It indicates that mechanical equipment has produced abnormal wear at this time, the particle size of the abrasive particles has been beyond than the normal size range, the device is not a normal working condition and immediately stops working, and we should take appropriate measures for the device to be checked.

Through the analysis of experimental results, we can obtain the trends of the abrasive particles' maximum and minimum particle size in the oil sample shown in Figure 6;

As you can be seen from the above figure, the maximum particle size of the abrasive particles in the oil sample increases with the use time of lubricants increasing, especially in the time period (779-860h) of the oil samples 3 and 4, the particle size of the abrasive particles change rapidly. It indicates that the internal parts of the transmission may occur abnormal wear and are in an abnormal 
state within this period of time. We should pay more attention and take measures to check the gearbox to prevent equipment failure caused by oil; the minimum size of the abrasive in oil remained unchanged, indicating that there has been normal wear gearbox and it is inevitable, but does not affect the normal operation of equipment.

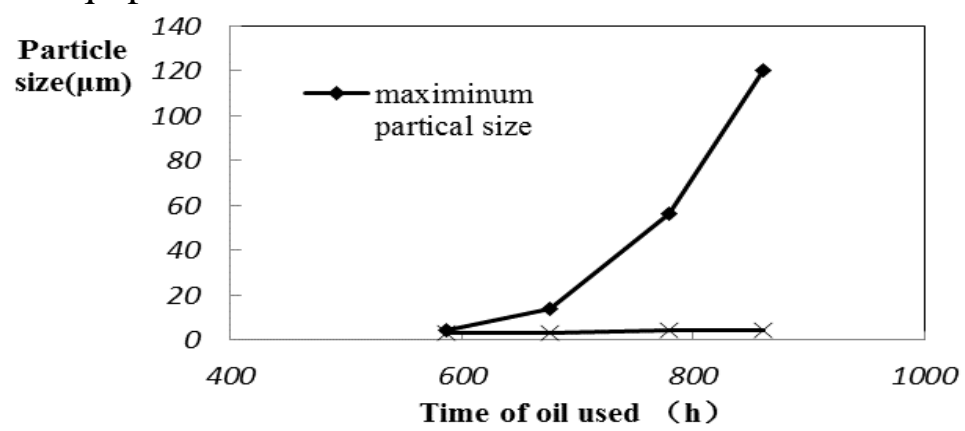

Fig.6 The trend of maximum and minimum partical size

\section{Conclusions}

Some experimental study can be made to monitor the number and size of abrasive particle in the oil of lubricant in SD16 bulldozer gearbox by the application of the laser particle size analysis method. One can determine the lubricant of gearbox to fine the abnormal wear of abrasive particles when it used the 800 hours and we need to check the equipment. On the other hand, we can detect the number and size of the abrasive particles in the oil quickly and efficiently, get the abrasive particles' particle size distribution at different times. It can provide the effective gist to judge the equipment running state. In short, laser particle size analysis as a new way to achieve oil monitoring can efficiently monitor the particle size distribution status of the abrasive particle in oil, and effectively prevent equipment operating faults, reduce unnecessary economic losses.

\section{Acknowledgements}

The authors wish to thank the financial support from National High Technology Research and Development Program (863 Program) (2013AA040204).

\section{References}

[1] Sun Zhiwei. Equipment monitoring and fault diagnosis method research based on oil analysis techniques [D]. Taiyuan University of Technology, 2012

[2] Tang Dexiu. Research of Ferrography technology for the automotive fault prediction [J]. Southwest University news (Natural Science), 2007,01:111-115.

[3] Zhou Xiaohong, Zhang Zhenyuan. The application of automatic particle counting method in jet fuel annex [J]. Mechanical and electronic, 2009, 08:73-75.

[4] Zheng Changsong, Ma Biao, Ma Yuan. Experimental study to monitoring Integrated Transmission state based on Oil Spectrum[J]. Spectroscopy and Spectral Analysis), 2009, 03: 749 $-751$.

[5] Jiang Liang. Malvern laser particle size analyzer used in oil research [J]. Modern Scientific Instruments, 2004, 06:48-50.

[6] Shu Xia, Wu Yucheng, Cheng Jigui, Xia Yonghong, Zheng Yuchun. Mastersizer 2000 laser particle size analyzer and its application [J]. Journal of he fei university of technology (natural science edition), 2007,02:164-167.

[7] Hu Hanxiang, Qiu Keqiang. Laser particle size analysis results in the application of morphology analysis [J]. Journal of physical and chemical inspection (physical volume), 2006,02:72-74.

[8] Zhu Zhaoju, Li Fangyi, Jia Xiujie, Li Guoyan, Wang Guangcun. Bulldozer gearbox failure diagnosis based oil monitoring technology [J]. Lubrication and sealing, 2014,07:83-87. 